\title{
Gene coexpression network analysis revealed biomarkers correlated with blast cells and survival in acute myeloid leukemia
}

\author{
YUMING PAN $^{1,2^{*}}$, QIAOXIA ZHANG ${ }^{2 *}$, XIAOPENG DENG ${ }^{2}$, NA AN $^{2}, \mathrm{XIN} \mathrm{DU}^{2}$ and JIAJUN LIU ${ }^{1}$ \\ ${ }^{1}$ Department of Hematology, The Third Affiliated Hospital of Sun Yat-Sen University, Guangzhou, Guangdong 510630; \\ ${ }^{2}$ Shenzhen Bone Marrow Transplantation Public Service Platform, Department of Hematology, \\ Shenzhen Second People's Hospital, The First Affiliated Hospital of Shenzhen University, \\ Shenzhen, Guangdong 518035, P.R. China
}

Received September 3, 2019; Accepted January 30, 2020

DOI: $10.3892 / \mathrm{mco} .2020 .2006$

\begin{abstract}
Acute myeloid leukemia (AML) is a hematological malignancy with a poorly understood pathogenesis, especially among patients with no known cytogenetic abnormalities. Furthermore, there is a lack of therapeutic gene targets and diagnostic biomarkers for the effective treatment of AML. The present study aimed to identify candidate biomarkers correlated with the clinical prognosis of patients with AML. Leukemic cells from 5 patients with AML exhibiting a normal karyotype, and hematopoietic cells from 5 healthy donors were processed for RNA sequencing (RNA-seq), and the obtained RNA expression profiles were subjected to weighted gene correlation network analysis. A novel group of genes (the red module) were identified to be significantly associated with AML, and this module contained a closely connected network with 147 nodes, which corresponded to 114 mRNAs. Analysis of the correlation between these mRNAs and blast cell percentage, overall survival (OS) and disease-free survival (DFS) using cases from The Cancer Genome Atlas (TCGA) database revealed that $C S F 3 R, A L P L$ and $L M T K 2$ were negatively associated with the percentage of blast cells, while high expression of these genes was associated with longer OS and DFS in patients with AML. The differential expression of these
\end{abstract}

Correspondence to: Dr Jiajun Liu, Department of Hematology, The Third Affiliated Hospital of Sun Yat-Sen University, Tianhe District, 600 Tianhe Road, Guangzhou, Guangdong 510630, P.R. China E-mail: jiajun.1@163.com

Dr Xin Du, Shenzhen Bone Marrow Transplantation Public Service Platform, Department of Hematology, Shenzhen Second People's Hospital, The First Affiliated Hospital of Shenzhen University, 3002 Sungang Road, Shenzhen, Guangdong 518035, P.R. China

E-mail: duxingz@medmail.com.cn

${ }^{*}$ Contributed equally

Key words: acute myeloid leukemia, weighted gene correlation network analysis, overall survival, disease-free survival, blast cells, prognosis three genes between patients with AML and healthy control subjects was supported using the Genotype-Tissue Expression and TCGA databases and was further confirmed using reverse transcription-quantitative (RT-qPCR). These genes exhibited significantly lower expression in patients with AML compared with control subjects. The results indicated that $C S F 3 R, A L P L$ and $L M T K 2$ exhibit the potential to be prognostic biomarkers. However, the biological functions of these three candidate genes need to be assessed in further studies.

\section{Introduction}

Acute myeloid leukemia (AML) is a hematologic malignancy characterized by aberrant clonal amplification of undifferentiated myeloid progenitors in bone marrow (BM) and results in dysregulated hematopoiesis (1). In the USA, the morbidity and mortality of AML are 13 and 7.1 per one hundred thousand persons, respectively (2), and these values in China are increasing (3). AML outcomes are classified as favorable, intermediate and adverse. Young patients with AML in the favorable and adverse groups had three-year overall survival (OS) rates of 66 and 12\% and three-year disease-free survival (DFS) rates of only 55 and $10 \%$, respectively $(4,5)$. Prognosis is related not only to age, sex, karyotype, white blood cell (WBCs) count and blast cell count but also to the expression and mutation of some critical genes (6). Several biomarkers have been proven to be useful in the diagnosis and prognosis of AML according to recent studies $(7,8)$ and some reports have shown that the expression levels of some genes, such as SETBPI, VEGFC and EVII, are associated with the risk level and the survival of patients (9-11). However, OS and DFS of patients with AML remain poor $(12,13)$. Therefore, identifying additional AML-related genes is urgently needed.

A typical feature of cancer is altered transcriptional networks originating from genetic aberrances, which drive disease occurrence and development $(14,15)$. These genetic abnormalities can act in conjunction with suitable upstream and downstream molecules to exert procarcinogenic activities. Therefore, the identification of novel transcriptional networks and key nodes should help combat abnormal transcription. Weighted gene correlation network analysis (WGCNA) is a statistical technique based on functions in the R software 
package and is used to identify groups of genes among microarray or transcriptome sequencing data that are highly correlated with each other and with biological traits (16). This biological analysis method has been used in pancreas, colon and bladder studies to identify important prognostic and therapeutic targets in data from the Gene Expression Omnibus (GEO) and The Cancer Genome Atlas (TCGA) (17-19). In this study, we used WGCNA to identify genes in the coexpressed network associated with AML.

\section{Materials and methods}

Clinical specimens. The clinical specimens used for RNA-seq were BM cells from five patients with de novo AML (three females and two males) with normal karyotypes. The five AML patients were adults aged 17, 24, 26, 31 and 44 years. The French-American and British (FAB) types were M1 (2), M4 (2) and M5 (1). The WBC count per liter of blood ranged from $106 \times 10^{9}$ to $642 \times 10^{9}$. Hematopoietic stem cells from mobilized peripheral blood (PB) of five healthy male donors with a mean age of 33.8 years were used as controls. The clinical specimens used to verify the differences in gene expression included samples from patients with AML (30) and healthy individuals (43). The thirty AML patients included 17 males and 13 females, and the average age was 41.1 years, ranging from 24 to 64 years old. Conventional cytogenetic analysis showed that 15 AML patients had a normal karyotype, while the karyotype of the other patients showed abnormalities. The FAB classification of the patients was stratified as follows: $1 \mathrm{M} 0,4 \mathrm{M} 1 \mathrm{~s}, 7 \mathrm{M} 2 \mathrm{~s}, 7 \mathrm{M} 4 \mathrm{~s}$ and $11 \mathrm{M} 5 \mathrm{~s}$. The healthy donors included 32 males and 11 females, and they ranged in age from 11 to 58 years, with a mean age of 33.6. The collection products from BM and $\mathrm{PB}$ were processes with red blood cell lysis buffer (Beijing Solarbio Science \& Technology Co., Ltd.) and the leukocytes remaining after centrifugation were used in subsequent RNA_seq or RT-qPCR.

This study protocol was approved by the institutional medical Ethics Committee of Shenzhen Second People's Hospital (Shenzhen, China). All patients and donors provided informed consent for the molecular analysis of their samples.

Online data resources. Clinicopathological data, including blast cells, OS, DFS and cytogenetic karyotype, of 200 AML specimens and the corresponding mRNA expression data were downloaded from the TCGA (https://gdc-portal.nci.nih. gov/). Because some data were invalid, the clinical data of 170 PB blast cells, 173 BM blast cells, 160 OS, 171 DFS and 156 karyotype samples were finally included to investigate the prognostic potential of candidate genes for AML. The mRNA expression data of whole blood from 337 healthy donors were downloaded from the Genotype-Tissue Expression (GTEx) database (https://gtexportal.org/home/index.html).

RNA sequencing. mRNA and IncRNA were isolated by removing the ribosomal RNA from total RNA. Then, the remaining RNA was fragmented (200-500 bp) and reverse transcribed into cDNA using random primers. A cDNA template with an adapter was used for fragment amplification and library construction. The libraries were sequenced using an Illumina HiSeq 2000 system (Total Genomics Solution Pte. Ltd.).
Clean reads were retrieved after filtering out sequences with poor quality and adaptor sequences from the raw reads and were aligned with the reference genome (UCSC hg 19) by HISAT (20).

Analysis of mRNA and lncRNA expression. The transcripts of the samples were reconstructed by StringTie (21), and redundant transcripts were eliminated using Cuffcompare software (22). The IncRNAs were collected through four filtering steps as follows: The short transcripts ( $<200 \mathrm{bp})$ were removed, the background transcripts were removed, the known transcripts and pre-mRNAs were removed, and the transcripts with protein-coding potential were removed. The number of reads mapped to the exon regions was calculated using HTseq software, and the expression levels of lncRNAs and mRNAs were calculated as the RPKM. CircRNA was selected as the intersection of the results, which were predicted by find_circ and CIRI software. The expression levels of the circRNAs were calculated with the pseudo RPKM method.

Weighted correlation network analysis. The mRNAs, lncRNAs and circRNAs were screened from the transcriptome profiles according to the following criteria: The expression levels of mRNAs and lncRNAs must be $\geq$ one in all specimens; the coefficient of variation must be at least 0.5 ; and circRNA must be expressed in $80 \%$ of the specimens. The resulting RNAs were used to construct the weighted gene coexpression network by WGCNA (https://labs.genetics.ucla.edu/horvath/htdocs/ CoexpressionNetwork/Rpackages/WGCNA/). First, Pearson's method was used to calculate the pairwise correlation coefficients of the genes and to construct the gene expressive correlation matrix. Next, the appropriate value of the soft-thresholding power $(\beta)$ was selected to build a weighted adjacency matrix, which was further transformed into a topological overlap matrix (TOM) and dissimilarity matrix. The latter was used for hierarchical clustering and dynamic cutting. The main modules were identified after an appropriate cutHeight point for cutting the tree was chosen and modules with similar eigengenes were merged.

Identifying the module associated with AML and functional enrichment analysis. The module eigengene (ME) represents a distinctive gene expression pattern of a module in a sample. The module-trait relationships were calculated using the correlation between modules' MEs and traits of AML. The gene significance (GS) was used to combine the clinical traits with the coexpression network. The higher the absolute value of GS, the more biologically meaningful the gene in a module is. Module significance (MS) is defined as the average absolute GS measured for all genes in a given module. The genes in the module of interest were subjected to Gene Ontology (GO) analyses. A P-value $<0.05$ was considered to be the cut-off criterion for significance.

Candidate prognostic target selection. The nodes (genes) in an undirected, weighted gene network corresponded to gene expression profiles. The edges between genes were determined by pairwise correlations between the expression levels of the genes. The genes in the module that were highly associated 
Table I. The optimal cut-off points of the candidate genes for OS and DFS.

\begin{tabular}{|c|c|c|c|c|}
\hline \multirow[b]{2}{*}{ Genes } & \multicolumn{2}{|c|}{ OS } & \multicolumn{2}{|c|}{ DFS } \\
\hline & $\begin{array}{c}\text { Cut-off } \\
\text { point }\end{array}$ & Statistic & $\begin{array}{c}\text { Cut-off } \\
\text { point }\end{array}$ & Statistic \\
\hline PTPRJ & 10.41 & 1.33 & 10.05 & 1.49 \\
\hline$W L S$ & 3.92 & 1.21 & 1.51 & 2.50 \\
\hline EXT1 & 4.20 & 3.04 & 7.00 & 1.85 \\
\hline KREMEN1 & 5.52 & 1.90 & 5.52 & 2.15 \\
\hline$A L P L$ & 0.94 & 1.89 & 3.30 & 3.57 \\
\hline$Q P C T$ & 2.22 & 2.46 & 6.31 & 1.17 \\
\hline CRI & 7.99 & 1.51 & 11.60 & 2.34 \\
\hline RASSF5 & 11.31 & 2.70 & 10.98 & 2.22 \\
\hline$R A B 43$ & 7.44 & 1.94 & 5.25 & 2.23 \\
\hline SEMA4B & 8.68 & 1.99 & 8.14 & 2.06 \\
\hline GLTID1 & 8.34 & 1.36 & 8.34 & 1.07 \\
\hline$S L C 25 A 37$ & 10.94 & 1.71 & 11.99 & 1.83 \\
\hline$P I K 3 C D$ & 12.46 & 1.73 & 12.46 & 1.99 \\
\hline LMTK2 & 9.34 & 3.29 & 9.34 & 2.99 \\
\hline IGSF6 & 5.69 & 1.80 & 5.69 & 1.52 \\
\hline ECE1 & 9.52 & 4.78 & 9.22 & 3.65 \\
\hline STEAP4 & 5.81 & 1.58 & 3.09 & 1.91 \\
\hline SLC $44 A 2$ & 11.09 & 3.12 & 10.95 & 2.49 \\
\hline$C S F 3 R$ & 14.50 & 4.14 & 13.43 & 4.31 \\
\hline$D O K 3$ & 11.80 & 1.24 & 10.52 & 2.51 \\
\hline
\end{tabular}

OS, overall survival; DFS, disease free survival.

with AML were selected as candidate genes with the criterion of a weighted value (edge width) that was not smaller than 0.4 between any two genes in the module. The weighted value between the genes was derived from the TOM matrix. We graphed the candidate gene coexpression network using Cytoscape software.

$R N A$ extraction and $R T-q P C R$. Clinical specimens were washed with RBC lysis buffer (Beijing Solarbio Science \& Technology Co., Ltd.) to remove the red blood cells (RBCs) and washed at least once with PBS buffer. Then, for the extraction of total RNA, the remaining white cells were suspended in RNAiso Plus reagent (Takara Bio, Inc.) and placed in a $-80^{\circ} \mathrm{C}$ refrigerator according to the manufacturer's instructions. A Prime Script II cDNA synthesis kit (Takara Bio, Inc.) was used to perform reverse transcription. In total, $2 \mu \mathrm{g}$ of RNA was converted into cDNA with random primers. The RT-qPCR system was prepared with TB Green Premix Ex Taq II (Takara Bio, Inc.) and the reaction was performed on a QuantStudio DX (Applied Biosystems; Thermo Fisher Scientific, Inc.). The primers for the candidate and reference genes are listed in Table SI.

Statistical analysis. The relationships between the percentage of blast cells in the BM or PB of AML patients and the expression levels of genes were statistically analyzed by
Pearson's correlation, and a two-tailed $\mathrm{P}<0.05$ was considered significant followed by Bonferroni multiple testing correction. The gene expression level was a continuous variable that was discretized for OS and DFS analyses. We determined the optimal cut-off point using the maximally selected rank statistics generated by R Version 3.5.0 (https://cran.r-project. org/web/packages/maxstat/index.html) (Table I). We compared the difference in survival between patients with high gene expression levels and patients with low gene expression levels by the log-rank test, and $\mathrm{P}<0.05$ indicated that the survival curves were significantly different.

\section{Results}

Construction of the modules by WGCNA among transcriptomes. The cDNA libraries of ten samples comprising leukemic cells from 5 AML patients with a normal karyotype and hematopoietic cells from 5 healthy donors were constructed for Illumina sequencing. A total of 1,022,008,940 clean reads with $153.3 \mathrm{~Gb}$ clean bases were obtained. The average Q20 and Q30 of the samples were $97.03 \%$ and 92.95, respectively (Table SII) and the top 20 differentially expressed genes (DEGs) are listed in Table SIII.

A total of 12,894 genes identified from RNA_seq were used to construct the gene coexpression network by WGCNA. The correlation coefficient matrix was calculated by Pearson's correlations among the 12,894 genes. Then, the adjacency matrix was constructed through index transformation, and the soft-thresholding power $(\beta)$ value was 10 according to the approximate scale-free topology criterion (Fig. 1A and B). A module is a group of genes with highly interconnected traits, as revealed by the topological overlap, and the modules were identified using hierarchical clustering dendrograms. Eighteen modules were obtained through the dynamic branch cutting method (cutHeight=0.18; Fig. 1C).

The red module was closely related to AML. Although six modules have significantly positive relationships with clinical traits and four modules have remarkably negative associations with the AML according to their correlation coefficients and P-values (Fig. 2A), we found that, among the eighteen modules, the red module had the greatest MS value (slightly higher than the firebrick4 module, Fig. 2B). This finding suggested that the red module may be the most biologically meaningful in AML. Furthermore, the protein-coding genes presented in each module were subjected to GO functional enrichment analysis. Assessment of the biological processes showed that the genes within the red module were enriched in processes associated with the biological characteristics of hematopoietic cells such as neutrophil activation $\left(\mathrm{P}\right.$-value $\left.=2.38 \times 10^{-45}\right)$, neutrophil degranulation $\left(\mathrm{P}\right.$-value $\left.=7.47 \times 10^{-43}\right)$ and leukocyte migration $\left(\mathrm{P}\right.$-value $=2.09 \times 10^{-18}$; Fig. $\left.3 \mathrm{~A}\right)$, while the genes in firebrick4 module were enriched in genes related to ribosome biogenesis $\left(\mathrm{P}\right.$-value $\left.=2.51 \times 10^{-12}\right)$ and ncRNA metabolic process ( $\mathrm{P}$-value $=3.14 \times 10^{-11}$; Fig. 3B); genes in mediumpurple3 were mainly enriched in $\mathrm{T}$ cell activation $\left(\mathrm{P}\right.$-value $=5.31 \times 10^{-27}$; Fig. 3C); and genes in the darkseagreen 4 were enriched in the regulation of the smoothened signaling pathway (P-value $=1.71 \times 10^{-5}$; Fig. 3D). The functional annotation of the genes in the red module revealed that they are 
A

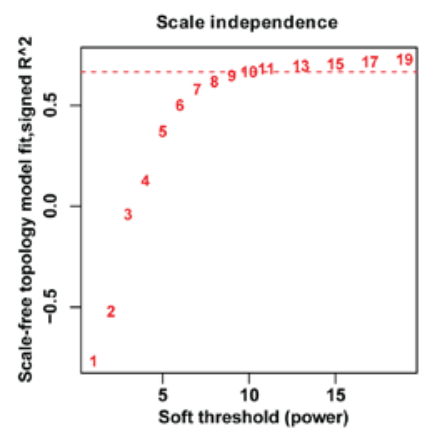

B

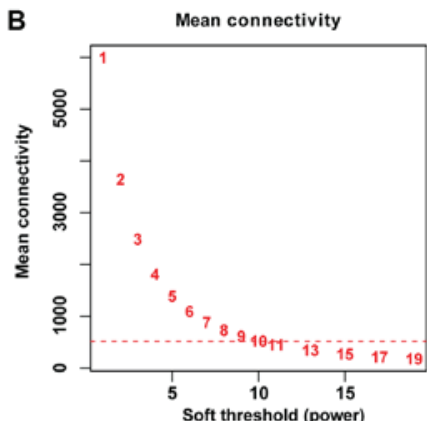

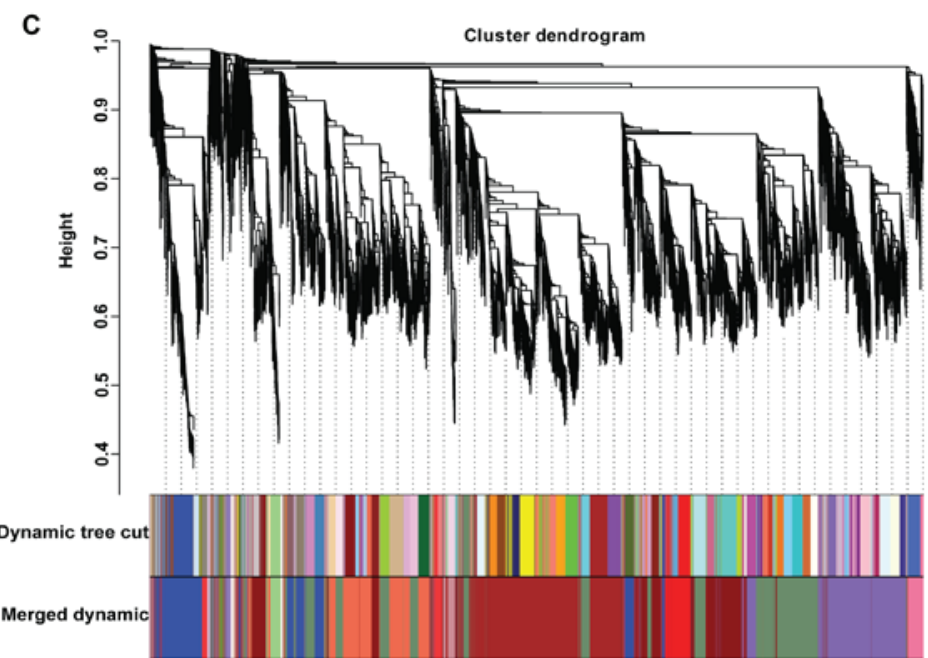

Figure 1. Modules were identified from the gene coexpression network using WGCNA. (A) The scale-free fitted curve shows the value of the degree of fitting according to $R^{2}$ based on the various soft threshold powers ( $\beta$ ). (B) The mean connectivity curve assisted in determining the value of $\beta$. (C) The modules were identified from the cluster dendrogram after dynamic branch cutting of the clustering tree to formed the original modules (cut Height=0.18) and merging of some modules based on the eigengene similarity of the original modules. WGCNA, Weighted gene correlation network analysis.

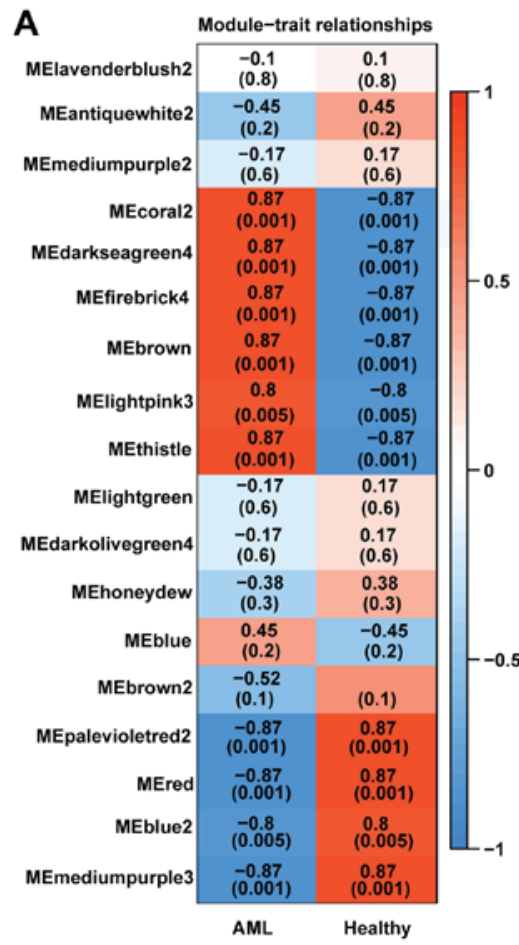

\section{B}

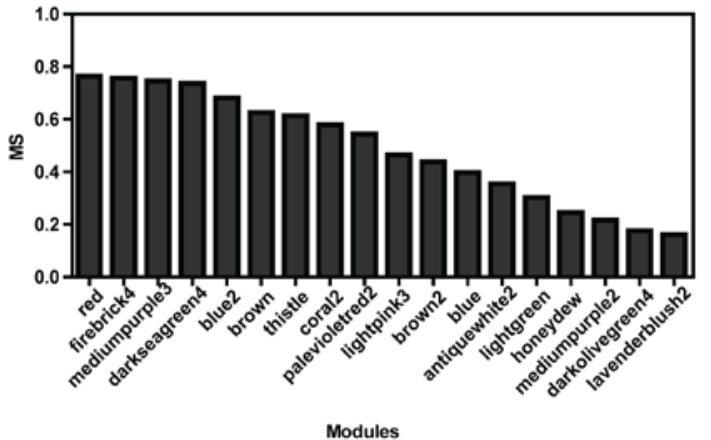

Figure 2. The genes in the red module were revealed to be negatively correlated with AML. (A) The heatmap displays the relationship between the ME of the modules and the traits; the values without parentheses are the correlation coefficients; red corresponds to a high correlation while blue indicates a low correlation; the values in parentheses are the P-values; $\mathrm{P}<0.05$ indicated a statistically significant difference. (B) The mean absolute value of GS (MS) of the eighteen modules; larger MS values correspond to the stronger relationships between the module and AML. AML, Acute myeloid leukemia; ME, module eigengene; GS, gene significance. 

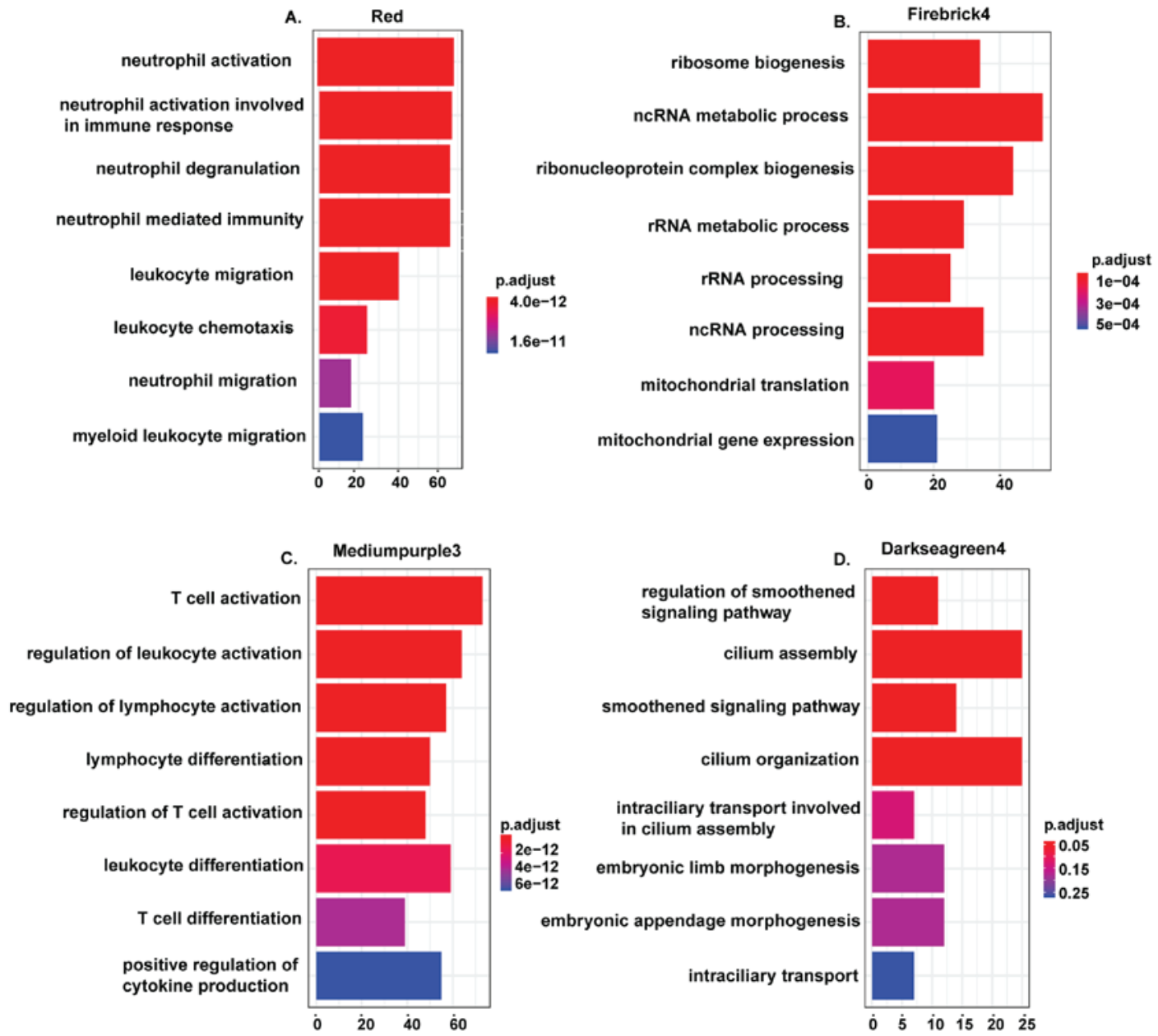

Figure 3. The genes from the red module were enriched in biological processes associated with the properties of hematopoietic cells. The biological processes for genes in the (A) red module, (B) firebrick4 module, (C) mediumpurple3 module and (D) darkseagreen4, (top 8). P<0.05 indicated a statistically significant difference.

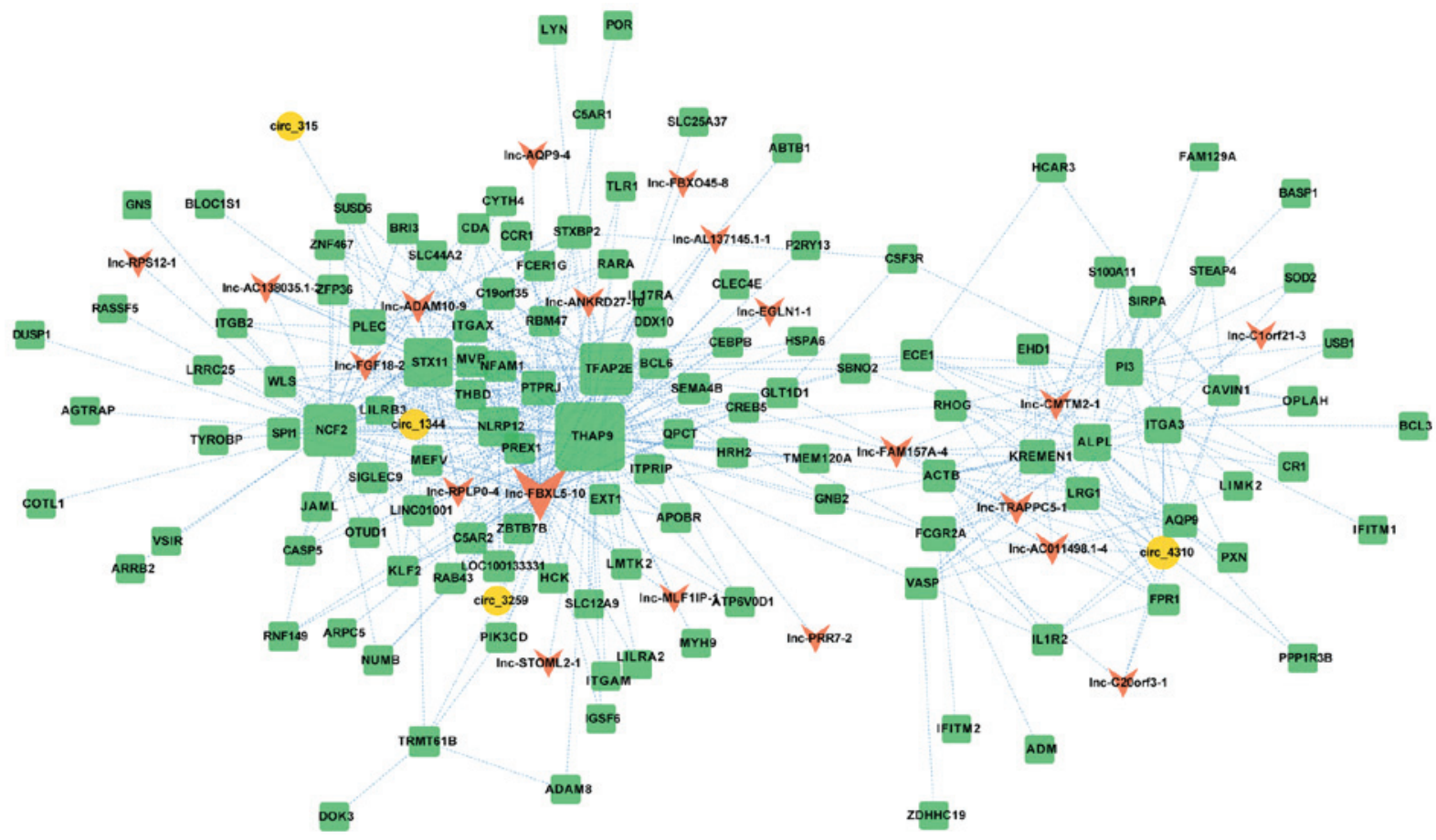

Figure 4. The gene coexpression network of the red module (edge $\geq 0.4$ ). Yellow dots indicate circRNAs, pink triangles indicate lncRNAs and green squares indicate mRNAs. The lines denote a coexpression relationship between the two points. The size of the point denotes the total connectivity value. 

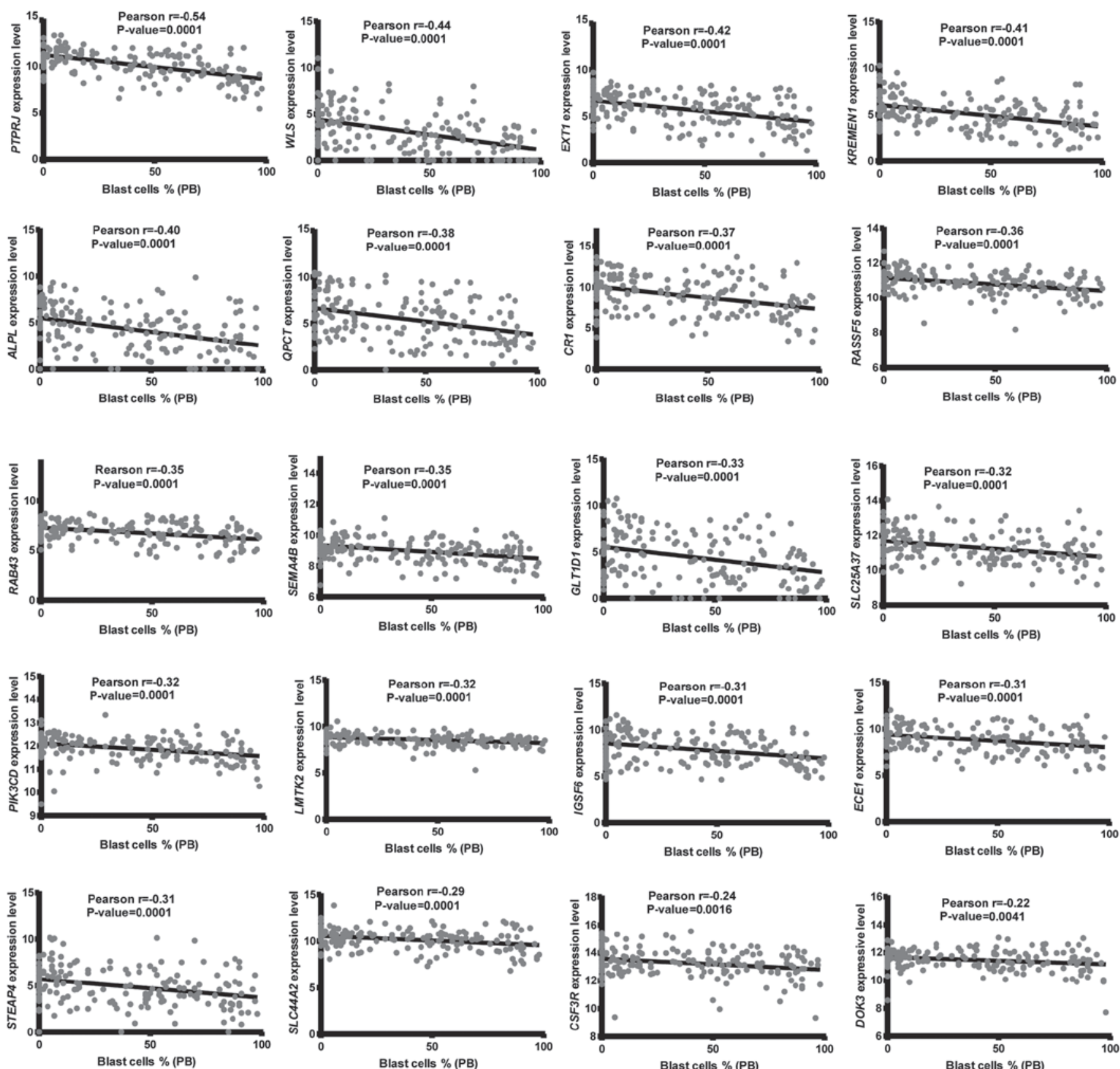

Figure 5. A total of 20 genes were indicated to be negatively correlated with the percentage of blast cells in $\mathrm{PB}, \mathrm{n}=170$. The correlations were calculated by Pearson's coefficient, and a two-tailed value of $\mathrm{P}<0.05$ indicated a statistically significant difference with Bonferroni's correction. $\mathrm{PB}$, peripheral blood.

intimately associated with the physiological development of hematopoietic cells and significantly correlated with AML. Based on the high pairwise-weighted values of genes in the red module, we drew the central nodes and their pattern of connectivity (Fig. 4). The network was composed of 147 nodes and 482 edges, corresponding to 123 mRNAs, 20 lncRNAs and 4 circRNAs (Table SIV).

Genes associated with the blast cells of AML patients. The circRNAs (4), IncRNAs (20), and some mRNAs (9) were removed from the 147 nodes in the network because of the lack of expression data on these RNAs in the TCGA database. The correlation between the expression levels of the remaining 114 genes and the blast cell percentage in BM (173) and PB (170) of patients diagnosed with AML were investigated. The results demonstrated that 23 genes had negative correlations with the percentage of blast cells in BM, although the relationships were weak (Pearson's $r$ value ranged from -0.33 to -0.15 ; Fig. S1). In addition, 20 of the 23 genes had a significant negative relationship with the percentage of blast cells in PB (Pearson's value is from -0.22 to -0.54 ; Fig. 5). These 20 genes were correlated with blast cells in both BM and PB and may play important roles in regulating the growth of leukemic cells.

Genes associated with OS and DFS of AML patients. Next, we used the endpoints of OS and DFS to analyze the association of the 20 genes with the survival of AML patients. The 

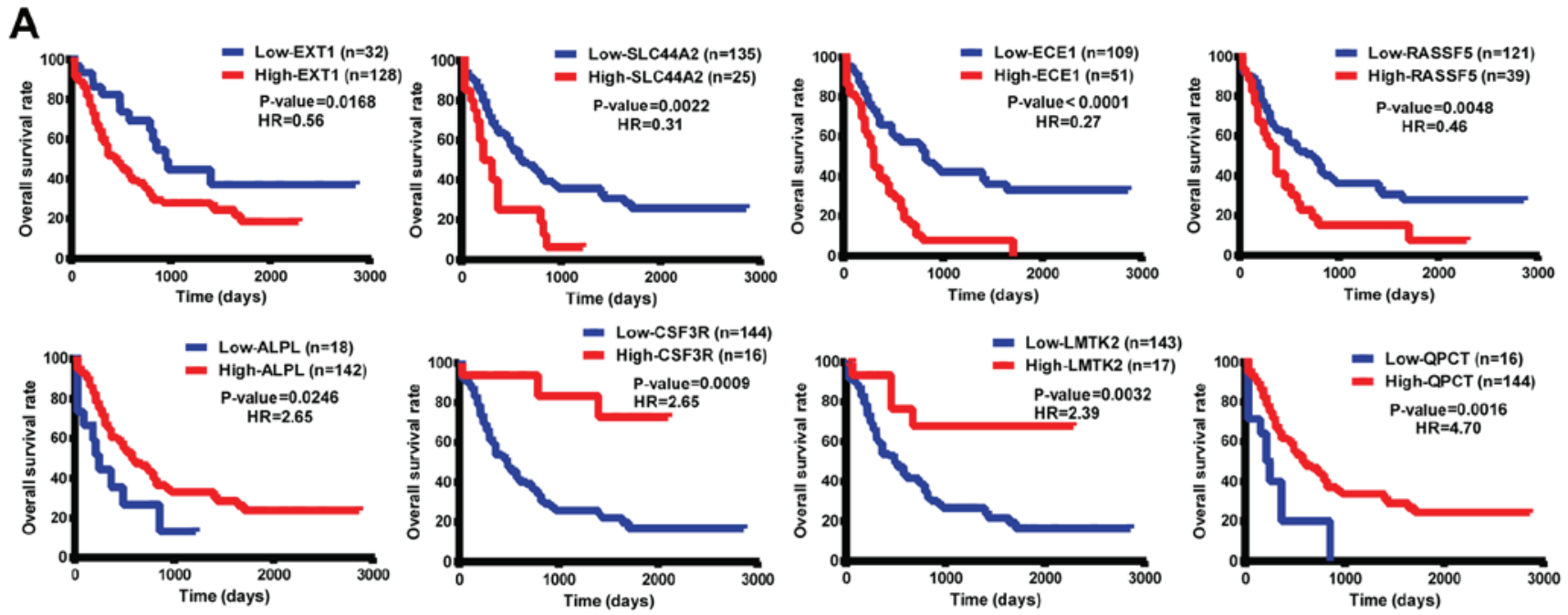

B
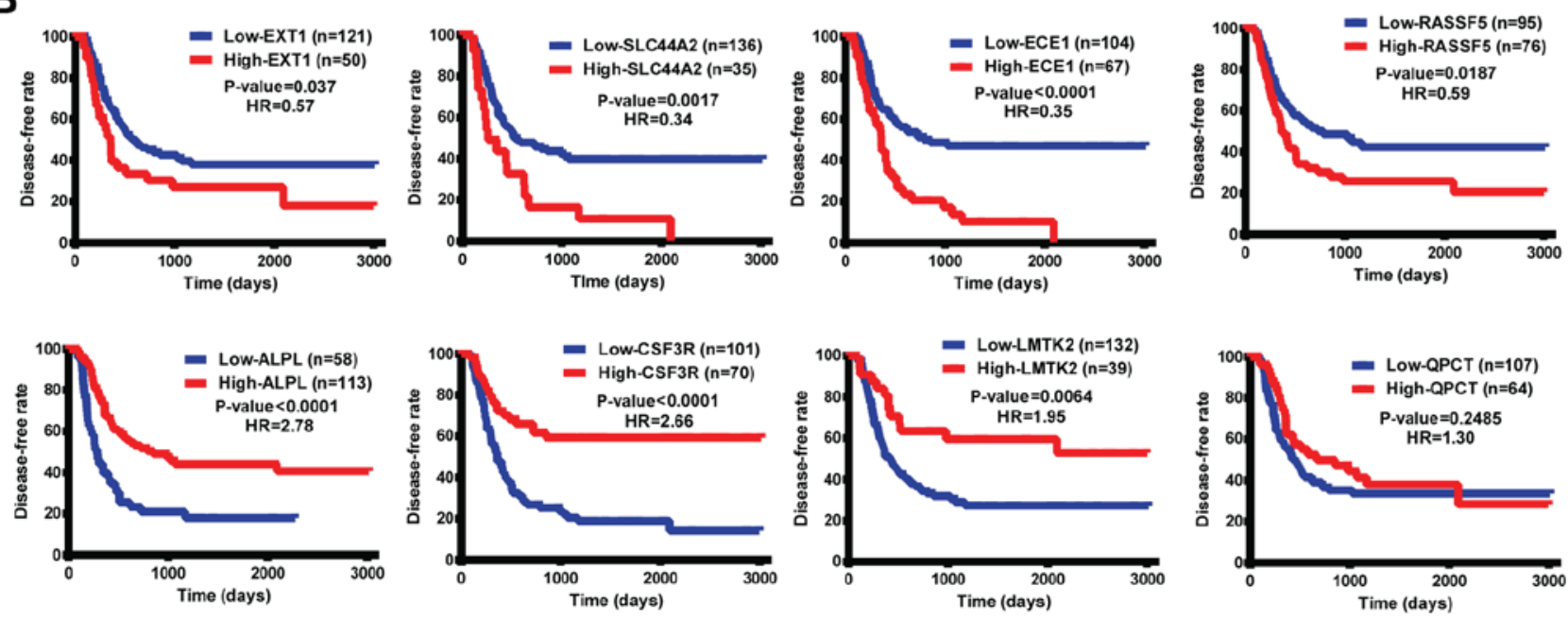

Figure 6. A total of eight genes were related to survival in patients with AML. (A) The genes associated with OS in patients with AML, n=160. (B) The genes related to DFS in patients with AML, $\mathrm{n}=171$. The cut-off point was determined in R with the tidyverse and survminer packages. The log-rank test was used to analyze the survival difference between the two groups and to determine the P-value ( $<0.05$ as significant) and the hazard ratio. AML, Acute myeloid leukemia; OS, overall survival; DFS, disease-free survival.

AML patients were categorized into low ( $\leq$ cut-off point) and high ( $>$ cut-off point) groups based on the expression levels of the 20 genes. Log-rank analysis showed that the OS of AML patients between the two groups was significantly different when the patients were stratified by the expression levels of the EXT1, SLC44A2, ALPL, CSF3R, ECE1, LMTK2, QPCT and $R A S S F 5$ genes. Patients with low expression levels of EXT1, $S L C 44 A 2, E C E 1$ and RASSF5 exhibited higher survival rates than those with high expression levels of these genes, while patients with high expression levels of $A L P L, C S F 3 R, L M T K 2$ and $Q P C T$ had better survival rates than those with low expression levels of these genes (Fig. 6A). In addition, all these genes (with the exception of $Q P C T$ ) were correlated with DFS (Fig. 6B).

Validation of the differences in expression levels of candidate genes between the AML and healthy groups. Our RNA-seq data showed that the expression levels of EXT1, SLC44A2, $A L P L, C S F 3 R, E C E 1, L M T K 2, Q P C T$, and RASSF5 were significantly lower in AML patients than in healthy controls (Fig. S2). More gene expression data of normal donors (337) and AML patients (156) were downloaded from the GTEx and TCGA databases to verify the differences in the expression levels of the genes of interest initially observed between the healthy and AML groups. This larger dataset also showed that the expression levels of these eight genes were significantly decreased in AML patients. In addition, CSF $3 R$ and SLC44A2 had remarkably reduced expression in the normal karyotypic group compared with the abnormal karyotypic group, while RASSF5 tended to have lower expression in AML patients with an abnormal karyotype (Fig. 7).

Further confirmation that the expression levels of CSF3R, $L M T K 2$ and ALPL were downregulated in AML patients compared with healthy controls was established using clinical samples (Fig. 8A-C). This result is logically consistent with the positive correlation of the three genes with OS and DFS in AML patients. Therefore, CSF3R, LMTK2 and ALPL show great potential as new prognostic markers of AML. 

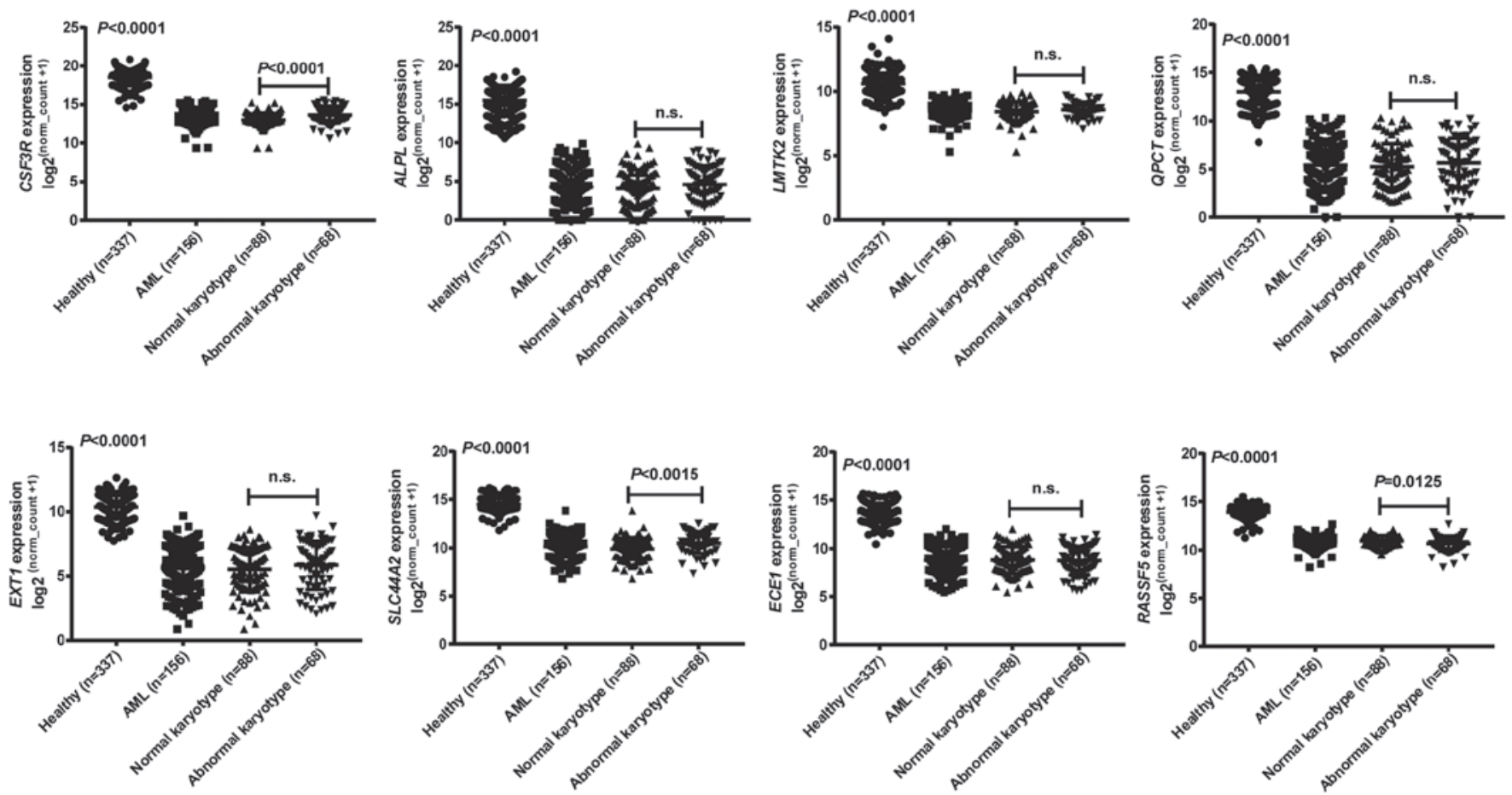

Figure 7. Differences in the expression levels of the candidate genes between the AML and healthy groups. The differentially expressed genes were verified using the GTEx (337) and TCGA (156) datasets. The AML group included 88 normal and 68 abnormal karyotypic samples. The expression levels of the genes were calculated by $\log 2\left(^{\left({ }^{\text {norm }} \_ \text {count }+1\right.}\right)$. Differences were tested for significance according to unpaired $t$ tests and one-way ANOVA in GraphPad Prism 5 software, and $\mathrm{P}<0.05$ indicated a statistically significant difference. GTEx, Genotype-Tissue Expression; TCGA, The Cancer Genome Atlas; AML, Acute myeloid leukemia.

A

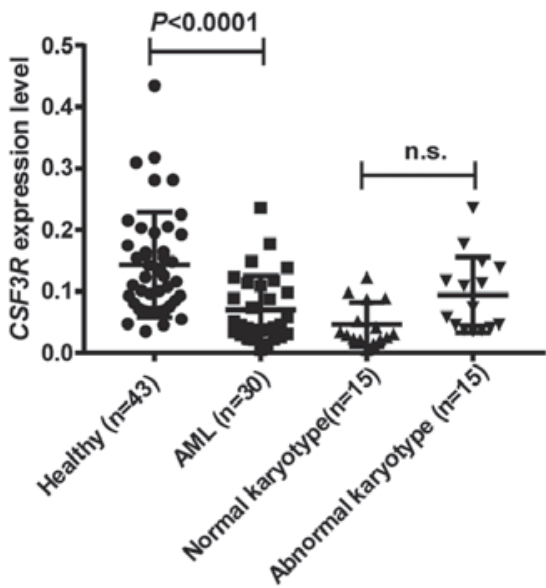

B

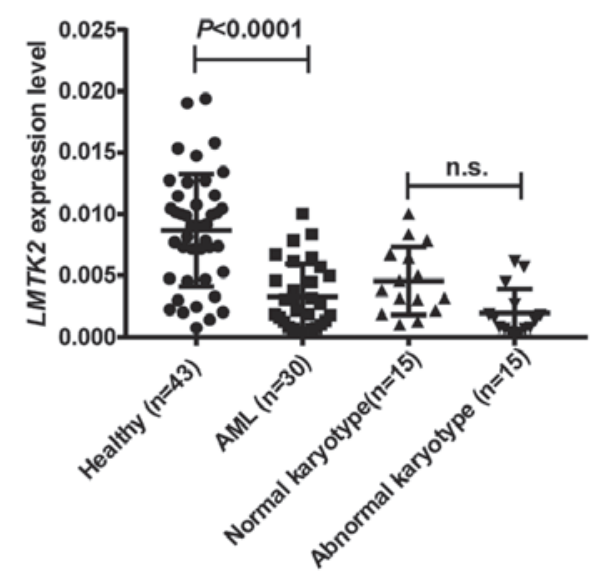

C

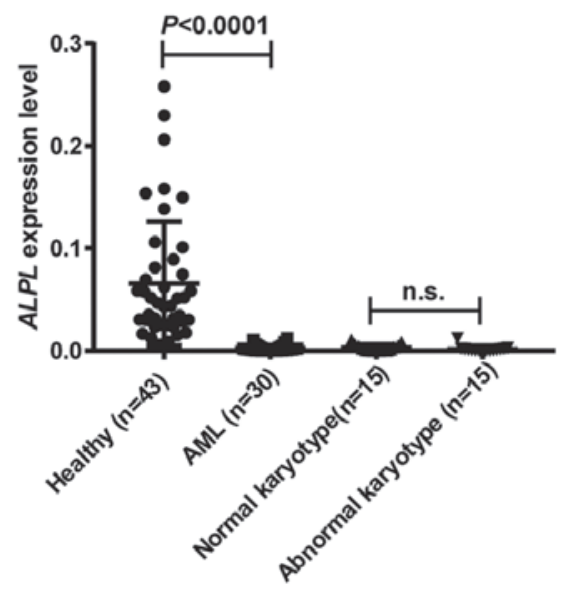

Figure 8. The relative expression levels of the three candidate genes in samples from the patients with AML and healthy donors were tested using reverse transcription-quantitative PCR. (A) CSF3R. (B) LMTK2. (C) ALPL. The GAPDH gene was used as a control for normalization of expression. The Tukey's test and one-way ANOVA were used to define the $\mathrm{P}$-value. $\mathrm{P}<0.05$ indicated a statistically significant difference.

\section{Discussion}

WGCNA is an efficient bioinformatics method used to reduce complicated transcriptomes into several gene modules with high interconnectivity and to determine the associations of these modules with clinical traits (16). In this way, we identified that the constructed red module was strongly negatively associated with AML, and the functional annotations revealed that the genes in the red modules are enriched in processes relating to neutrophils activation, neutrophil degranulation and leukocyte migration. Through a series of correlation analyses, we found that three genes with anomalous low expression levels were significantly inversely correlated with the percentage of blast cells but positively correlated with the survival of AML patients.

The three genes $C S F 3 R, A L P L$, and $L M T K 2$ had extremely downregulated expression levels in AML patients compared with healthy controls, and the downregulation of these genes was associated with worse OS and DFS in AML patients. Receptor for colony stimulating factor 3 (CSF3R) is well 
known to regulate the production, differentiation, and function of granulocytes (23). Mutations in this gene are frequently present in patients with chronic neutrophilic leukemia (CNL) and can be used as accurate diagnostic markers for CNL (24). Mutations in CSF3R are rare in AML and have been reported to highly overlap with CEBP $\alpha$ mutations in AML patients, which predicts a poor outcome $(25,26)$. Our data show that $C S F 3 R$ is tended to underexpressed in AML patients with a normal karyotype and may serve as a special genetic biomarker for the prognosis and treatment of AML patients with a normal karyotype. Tissue-nonspecific alkaline phosphatase (ALPL) plays a role in bone biomineralization, and mutations in this gene are used to diagnose hypophosphatasia (27). Further studies are needed to reveal the functions of ALPL in AML. Lemur tyrosine kinase 2 (LMTK2) is a tumor suppressor that is downregulated in some neurodegenerative diseases (28) and can inhibit the activity of PP1C by controlling GSK3 $\beta$ phosphorylation (29). The effect of LMTK2 on the pathogenesis of AML has not been studied, but LMTK2 is predicted to enhance the cytotoxic activity of natural killer cells to kill leukemic blast cells via inhibition of GSK3 $\beta$ (30).

In the present study, we using RNA_seq combined with WGCNA statistical method finding CSF3R, ALPL and LMTK2 are potential prognostic markers for AML but need to be studied more thoroughly to confirm their biological functions in this disease. However, the limitation of RNA_seq is the result simply represents the mean expression of genes in white blood cells which contain diverse cell populations (31). The newly developed single-cell RNA sequencing can compensate for the defect and provide more huge and accurate data. The latest method would help in finding exceptional subpopulations and genes of interest in the future.

\section{Acknowledgements}

Not applicable.

\section{Funding}

The present study work was supported by grants from the National Natural Science Foundation of China (grant no. 81350027), the Major Projects of the Guangdong Natural Team (grant no. 2014A030312012) and the Clinic Research Program of Shenzhen Second People's Hospital (grant no. 20173357201802).

\section{Availability of data and materials}

The RNA_seq data from clinical samples were analyzed in the current study and are available in the public repository in the NCBI database (SRA accession: PRJNA576718). The data used to confirm this research are available from TCGA (https://gdc-portal.nci.nih.gov/) and GTEx (https://gtexportal. org/home/index.html).

\section{Authors' contributions}

JL, QZ and XD contributed to design and supervision of the project. YP and QZ were responsible for writing the manuscript. YP and XD analyzed the RNA data using WGCNA and NA contributed to the collection of the clinical specimens. All authors have read and approved the final version of this manuscript.

\section{Ethics approval and consent to participate}

This study protocol was approved by the institutional medical Ethics Committee of Shenzhen Second People's Hospital (Shenzhen, China). All patients and donors provided informed consent for the molecular analysis of their samples.

\section{Patient consent for publication}

Not applicable.

\section{Competing interests}

The authors declare that they have no competing interests.

\section{References}

1. Estey EH: Acute myeloid leukemia: 2013 update on risk-stratification and management. Am J Hematol 88: 318-327, 2013.

2. Desai P, Hassane D and Roboz GJ: Clonal hematopoiesis and risk of acute myeloid leukemia. Best Pract Res Clin Haematol 32: 177-185, 2019.

3. Chang KH, Hwang WL, Muo CH, Hsu CY and Teng CJ: Outcome and late effects among acute myeloid leukemia survivors: A nationwide population-based study. Support Care Cancer 24: 4993-5000, 2016.

4. Dohner H, Estey E, Grimwade D, Amadori S, Appelbaum FR, Büchner T, Dombret H, Ebert BL, Fenaux P and Larson RA: Diagnosis and management of AML in adults: 2017 ELN recommendations from an international expert panel. Blood 129: 424-447, 2017.

5. Mrozek K, Marcucci G, Nicolet D, Maharry KS, Becker H, Whitman SP, Metzeler KH, Schwind S, Wu YZ and Kohlschmidt J: Prognostic significance of the European LeukemiaNet standardized system for reporting cytogenetic and molecular alterations in adults with acute myeloid leukemia. J Clin Oncol 30: 4515-4523, 2012.

6. Deschler B and Lübbert M: Acute myeloid leukemia: Epidemiology and etiology. Cancer 107: 2099-2107, 2006.

7. Junge A, Bacher U, Mueller BU, Keller P, Solenthaler M and Pabst T: Adverse outcome of AML with aberrant CD16 and CD56 NK cell marker expression. Hematol Oncol: Jun 3, 2018 (Epub ahead of print).

8. Zahran AM, Mohammed Saleh MF, Sayed MM, Rayan A Ali AM and Hetta HF: Up-regulation of regulatory T cells, CD200 and TIM3 expression in cytogenetically normal acute myeloid leukemia. Cancer Biomark 22: 587-595, 2018.

9. Cristobal I, Blanco FJ, Garcia-Orti L, Marcotegui N, Vicente C, Rifon J, Novo FJ, Bandres E, Calasanz MJ, Bernabeu C and Odero MD: SETBP1 overexpression is a novel leukemogenic mechanism that predicts adverse outcome in elderly patients with acute myeloid leukemia. Blood 115: 615-625, 2010

10. de Jonge HJ, Valk PJ, Veeger NJ, ter Elst A, den Boer ML, Cloos J, de Haas V, van den Heuvel-Eibrink MM, Kaspers GJ and Zwaan CM: High VEGFC expression is associated with unique gene expression profiles and predicts adverse prognosis in pediatric and adult acute myeloid leukemia. Blood 116: 1747-1754, 2010.

11. Groschel S, Lugthart S, Schlenk RF, Valk PJ, Eiwen K, Goudswaard C, van Putten WJ, Kayser S, Verdonck LF and Lübbert M: High EVI1 expression predicts outcome in younger adult patients with acute myeloid leukemia and is associated with distinct cytogenetic abnormalities. J Clin Oncol 28: 2101-2107, 2010.

12. Vasu S, Kohlschmidt J, Mrozek K, Eisfeld AK, Nicolet D, Sterling LJ, Becker H, Metzeler KH, Papaioannou D and Powell BL: Ten-year outcome of patients with acute myeloid leukemia not treated with allogeneic transplantation in first complete remission. Blood Adv 2: 1645-1650, 2018. 
13. Dohner H, Estey EH, Amadori S, Appelbaum FR, Buchner T, Burnett AK, Dombret H, Fenaux P, Grimwade D and Larson RA: Diagnosis and management of acute myeloid leukemia in adults: Recommendations from an international expert panel, on behalf of the European LeukemiaNet. Blood 115: 453-474, 2010.

14. Assi SA, Bonifer C and Cockerill PN: Rewiring of the transcription factor network in acute myeloid leukemia. Cancer Inform 18: $1176935119859863,2019$.

15. Thoms JAI, Beck D and Pimanda JE: Transcriptional networks in acute myeloid leukemia. Genes Chromosomes Cancer 58: 859-874, 2019

16. Langfelder $\mathrm{P}$ and Horvath S: WGCNA: An R package for weighted correlation network analysis. BMC Bioinformatics 9: $559,2008$.

17. Zhou Z, Cheng Y, Jiang Y, Liu S, Zhang M, Liu J and Zhao Q: Ten hub genes associated with progression and prognosis of pancreatic carcinoma identified by co-expression analysis. Int J Biol Sci 14: 124-136, 2018.

18. Liu R, Zhang W, Liu ZQ and Zhou HH: Associating transcriptional modules with colon cancer survival through weighted gene Co-expression network analysis. BMC Genomics 18: 361, 2017.

19. Li S, Liu X, Liu T, Meng X, Yin X, Fang C, Huang D, Cao Y, Weng $H$, Zeng $X$ and Wang $X$ : Identification of biomarkers correlated with the TNM staging and overall survival of patients with bladder cancer. Front Physiol 8: 947, 2017.

20. Kim D, Langmead B and Salzberg SL: HISAT: A fast spliced aligner with low memory requirements. Nat Methods 12: 357-360, 2015.

21. Pertea M, Pertea GM, Antonescu CM, Chang TC, Mendell JT and Salzberg SL: StringTie enables improved reconstruction of a transcriptome from RNA-seq reads. Nat Biotechnol 33: 290-295, 2015.

22. Trapnell C, Roberts A, Goff L, Pertea G, Kim D, Kelley DR, Pimentel H, Salzberg SL, Rinn JL and Pachter L: Differential gene and transcript expression analysis of RNA-seq experiments with TopHat and Cufflinks. Nat Protoc 7: 562-578, 2012.

23. Touw IP and van de Geijn GJ: Granulocyte colony-stimulating factor and its receptor in normal myeloid cell development, leukemia and related blood cell disorders. Front Biosci 12: 800-815, 2007.
24. Elliott MA and Tefferi A: Chronic neutrophilic leukemia: 2018 update on diagnosis, molecular genetics and management. Am J Hematol 93: 578-587, 2018.

25. Maxson JE, Ries RE, Wang YC, Gerbing RB, Kolb EA, Thompson SL, Guidry Auvil JM, Marra MA, Ma Y and Zong Z: CSF3R mutations have a high degree of overlap with CEBPA mutations in pediatric AML. Blood 127: 3094-3098, 2016.

26. Su L, Gao S, Tan Y, Lin H, Liu X, Liu S, Yang Y, Sun J and Li W: CSF3R mutations were associated with an unfavorable prognosis in patients with acute myeloid leukemia with CEBPA double mutations. Ann Hematol 98: 1641-1646, 2019.

27. Tenorio J, Alvarez I, Riancho-Zarrabeitia L, Martos-Moreno GA, Mandrile G, de la Flor Crespo M, Sukchev M, Sherif M, Kramer I and Darnaude-Ortiz MT: Molecular and clinical analysis of ALPL in a cohort of patients with suspicion of Hypophosphatasia. Am J Med Genet A 173: 601-610, 2017.

28. Bencze J, Morotz GM, Seo W, Bencs V, Kalman J, Miller CCJ and Hortobágyi T: Biological function of Lemur tyrosine kinase 2 (LMTK2): Implications in neurodegeneration. Mol Brain 11: 20, 2018.

29. Conti A, Majorini MT, Fontanella E, Bardelli A, Giacca M, Delia D, Mano M and Lecis D: Lemur tyrosine kinase 2 (LMTK2) is a determinant of cell sensitivity to apoptosis by regulating the levels of the BCL2 family members. Cancer Lett 389: 59-69, 2017.

30. Parameswaran R, Ramakrishnan P, Moreton SA, Xia Z, Hou Y, Lee DA, Gupta K, deLima M, Beck RC and Wald DN: Repression of GSK3 restores NK cell cytotoxicity in AML patients. Nat Commu 7: 11154, 2016.

31. van Galen P, Hovestadt V, Wadsworth Ii MH, Hughes TK, Griffin GK, Battaglia S, Verga JA, Stephansky J, Pastika TJ and Lombardi Story J: Single-cell RNA-seq reveals AML hierarchies relevant to disease progression and immunity. Cell 176: 1265-81.e24, 2019. 